\title{
Start in ein nachhaltiges Jahrtausend
}

Mit langer Verzögerung setzt die Bundesregierung nun um, was Experten schon lange fordern: Eine nationale Nachhaltigkeitsstrategie, die die Aktivitäten bündelt und vorantreibt. Ein Motor dieses Prozesses wird der nev eingerichtete Nachhaltigkeitsrat sein. Die Umsetzung in das politische Alltagshandeln soll ein "Green Cabinet" aus hierfür zuständigen Staatssekretären gewährleisten.

$\mathrm{P}$ Von Marion Caspers-Merk olitische Weichen für die Zukunft zu stellen, gerät im politischen Alltag gerne unter die Räder. So geschehen 1992, als in Rio allen Teilnehmerstaaten die Hausaufgabe erteilt wurde, eine nationale Nachhaltigkeitsstrategie $\mathrm{zu}$ erarbeiten. Ungenügend würde man in der Schule eine Leistung beurteilen, die nicht erbracht wurde. Also ungenügend für Deutschland, denn bislang ging in Deutschland im Vergleich zu anderen europäischen Staaten die Entwicklung einer nationalen Nachhaltigkeitsstrategie nur schwer voran (1). Auch die alte Bundesregierung kam über Ansä̈ze nicht hinaus, einzelne Initiativen im Umweltministerium fanden nie den Weg ins Kabinett. Kurz: Projektorientiertes, vernetztes Arbeiten, wie in vielen Bereichen bereits selbstverständlich praktiziert, funktionierte bislang nicht, Ressortdenken von Ministerialbürokratien und zu viele verschiedene Beratungsgremien verhinderten die zügige Erarbeitung einer Nachhaltigkeitsstrategie.

Nachhaltige Entwicklung bedeutet, nicht auf Kosten künftiger Generationen und anderer Erdteile zu leben. Nachhaltigkeit heißt, in einem umfassenden Sinn Vorsorge zu treffen für unsere Kinder und Enkel. Insoweit verlangt Nachhaltigkeit nicht allein die Bewahrung unserer natürlichen Lebensgrundlagen. Auch solide Staatsfinanzen, gerechte Sozialversicherungssysteme, Investitionen in Bildung und Forschung sind Gebote nachhaltiger Entwicklung. Der Ausgangspunkt der Nachhaltigkeitsdebatte bleibt jedoch die Gefährdung der ökologischen Kreisläufe im globalen Maßstab.

Die Dreidimensionalität der Nachhaltigkeit aus Ökologie, Ökonomie und Sozialem erfordert, dass diese Belange zusammengeführt werden. Dabei erfordert die Ökologie unser Hauptinteresse, denn alles Wirtschaften und damit auch die soziale Wohlfahrt stehen letztendlich unter dem Vorbehalt unserer natürlichen Lebensgrundlagen.
Die Nachhaltigkeitsstrategie ist ein Instrument, um einen umfassenden Reformprozess der ökologischen Modernisierung einzuleiten und $\mathrm{zu}$ unterstiitzen, der alle Bereiche von Politik und Gesellschaft mit einbezieht. Das gelingt nur, wenn wir dies als Querschnittsaufgabe verstehen, die alle Bereiche der Regierungsarbeit durchdringt und von einem breiten gesellschaftlichen Konsens getragen wird. Dabei müssen Handlungsnotwendigkeiten transparent gemacht, Schwerpunkte gesetzt und konkrete Ziele formuliert werden.

\section{- Umfassender Reformprozess}

Die Bundesregierung erarbeitet eine nationale Nachhaltigkeitsstrategie, die sich vor allem mit folgenden Bereichen beschäftigten wird:

- Integrierte Lösungskonzepte im Klimaschutz wie Erhöhung der Energieeffizienz und innovative Lösungen für Energieeinsparung in allen Nutzungsbereichen,

- Ressourcenschonung und -management wie schonender und sparender Umgang mit Wasser, fossilen Energieträgern, Böden und Naturgütern, Kreislaufwirtschaft und Stoffstrommanagement,

- Integrierte Lösungskonzepte im Verkehrsbereich wie die Verlagerung von Verkehr, Stadt der kurzen Wege, technische Optimierung von Verkehrslösungen,

- Konsumverhalten und Umweltbildung wie die Förderung umweltgerechter Verhaltensmuster und

- Forschung und Entwicklung wie innovative Lösungen für integrierten Umweltschutz.

Eine zentrale Rolle bei der Erarbeitung der nationalen Nachhaltigkeitsstrategie ist dabei dem einzurichtenden Rat für nachhaltige Entwicklung zugedacht. Er soll die Bundesregierung beim Prozess der Umsetzung maßgebend unterstïtzen und vor allem folgende Aufgaben wahrnehmen:

- Beratung der Politik im Vorfeld staatlicher Maßnahmen,
Begleitung der Umsetzung von Zielen und Maßnahmen nachhaltiger Entwicklung,

- Vertiefung des Dialogs zwischen den staatlichen und gesellschaftlichen Akteuren und

- Stärkung des gesellschaftlichen Konsenses über das Leitbild der nachhaltigen Entwicklung und Maßnahmen zu seiner Umsetzung.

Dieses Gremium soll hochrangig mit Persönlichkeiten aus Wirtschaft, Wissenschaft, Politik und Gesellschaft besetzt sein, die nicht aufgrund ihrer Verbandsfunktion, sondern aufgrund ihres persönlichen Engagements Mitglied werden. Die Mitgliederzahl sollte fünfzehn Personen nicht übersteigen, um durch eine schlank gehaltene Struktur eine effektive Arbeitsweise zu gewährleisten. Um der politischen Bedeutung Ausdruck zu verleihen, wird der Nachhaltigkeitsrat beim Kanzleramt angesiedelt sein.

Parallel dazu hat die Bundesregierung nach britischem Vorbild ein "Green Cabinet“ der nachhaltigkeitsrelevanten Ressorts eingerichtet, um die laufende Regierungspolitik auf ihre Nachhaltigkeitswirkung zu überprüfen. Zudem soll der Prozess der Erarbeitung einer Nachhaltigkeitsstrategie in regelmäßigen Abständen eine intensive parlamentarische Begleitung, Beteiligung und Beachtung durch Bundestag und Bundesrat erfahren.

Die deutsche Politik braucht grundlegende Reformen, auch in den Strukturen. Wir haben zu wenig Beteiligung und zu wenig Querschnitt. Hier kann und wird der Nachhaltigkeitsrat Abhilfe schaffen: Besetzt mit Sachverständigen und ausgestattet mit einem Selbstbefassungsrecht, wird er auch dazu beitragen, einzelfallbezogenes Denken zu überwinden, um endlich wieder einen Blick für die Zusammenhänge zu gewinnen und den Weg in ein zukunftsverträgliches, nachhaltiges Jahrtausend zu ebnen.

Anmerkung

(1) Vgl. hierzu Rüling, Anneli/ Volkery, Axel: Umweltplanung ante Portas?

In: Ökologisches Wirtschaften 3/99, S. 8-9.

\section{Die Autorin}

Marion Caspers-Merk ist für die SPD Mitglied des Deutschen Bundestages und war in der letzten Legislaturperiode Vorsitzende der EnqueteKommission "Schutz des Menschen und der Umwelt". Kontakt: Platz der Republik, 11011 Berlin, Tel. 030/ 227-75783, Fax -76613,

E-mail: marion.caspers-merk@bundestag.de 
(c) 20I0 Authors; licensee IÖW and oekom verlag. This is an article distributed under the terms of the Creative Commons Attribution Non-Commercial No Derivates License (http://creativecommons.org/licenses/by-nc-nd/3.o/), which permits unrestricted use, distribution, and reproduction in any medium, provided the original work is properly cited. 